\title{
A Feedback Controller for Biped Humanoids that Can Counteract Large Perturbations During Gait
}

\author{
Taku Komura and Howard Leung \\ City University of Hong Kong \\ taku@ieee.org,howard@cityu.edu.hk
}

\author{
Shunsuke Kudoh \\ The University of Tokyo \\ kudoh@cvl.iis.u-tokyo.ac.jp
}

\author{
James Kuffner \\ Carnegie Mellon University \\ kuffner@cs.cmu.edu
}

\begin{abstract}
In this paper, we propose a new method for biped humanoids to compensate for large amounts of angular momentum induced by strong external perturbations applied to the body during gait motion. Such angular momentum can easily cause the humanoid to fall down onto the ground. We use an Angular Momentum inducing inverted Pendulum Model (AMPM), which is an enhanced version of the 3D linear inverted pendulum model to model the robot dynamics. Because the AMPM allows us to explicitly calculate the angular momentum generated by the ground reaction force, it is possible to calculate a counteracting motion that compensates for the angular momentum generated by external perturbations in realtime.
\end{abstract}

\section{INTRODUCTION}

Research related to biped locomotion and stable walking control of humanoid robots has advanced rapidly in recent years. Several researchers have proposed feed-forward algorithms for humanoid walking based on ZMP criteria [6], [3], [1], [5], [2], [9], [11]. The trajectories generated by these methods are model-based and result in dynamically stable motions provided that the trajectory of the Zero Moment Point lies inside the convex hull of the area of support.

Gaited motion by bipeds can be quite sensitive to modeling errors and uncertainty. In order to achieve stable gaits, a feedback controller typically must be used. Kajita et al.[7] calculated the additional rotational momentum that must be applied to the body to maintain balance by using the angular momentum as a direct reference. Napoleon et al.[10] proposed a feedback method based on a two link inverted pendulum model. In this research, the motion generated by the motion planner are kept the same, and the feedback controller tries to reduce the difference between the current state and the ideal motion. This feedback controller was used to simulate stable standing and kicking motions.

Humans, on the other hand, use a variety of complex strategies in order to maintain balance according to the amount of force applied to the body. For example, for relatively weak perturbations, the impact is absorbed by the ankle joint; the posture of the upper part of the body remains unchanged. When the impact is larger, the hip and knee joints are used, and the whole body is used to absorb the impact. If the impact is even stronger, the human will alter their gait and step out one or two steps to counteract the additional linear and angular momentum. Evidence from biomechanical studies indicate that a number of different strategies are prepared in advance, and the most appropriate response motion is launched when the perturbation occurs. This means that overall balance is preserved not only through feedback control, but the feedforward motion is also changed according to the current state of the body. These two balance compensation strategies vastly increase the flexibility and robustness of the human gait. In order to enable biped humanoids to approach the same level of performance and stability as humans, a balancing controller that is capable of "retuning" the upcoming balancing motion in real-time is needed.

In this paper, we propose a new feedback method for biped humanoids to counteract strong external perturbation using the Angular Momentum inducing inverted Pendulum Model (AMPM), which is an enhanced version of the 3D Linear Inverted Pendulum Mode (3DLIPM). Using AMPM, it is possible to explicitly calculate the angular momentum generated during the motion. After the perturbation, new trajectories of the desired COM and the angular momentum are calculated to compensate for the increased angular momentum due to the disturbance. By using these trajectories as constraints, the trajectories of the generalized coordinates including the position of the center of the hips and the joint angles that satisfy those constraints are calculated using inverse kinematics, as done in [8], [4].

We also propose a new criteria called the difference of inertia, that is based on the difference of the moment of inertia between the current posture and the corresponding posture in the original motion. By using the difference of inertia as a criteria, the angular momentum needed to bring the posture back to the original motion can be estimated. As a result, it is possible to calculate the motion of the humanoid which counteracts the external perturbation, and then gradually moves back to the original gait motion. A number of experiments were conducted to check the validity of the proposed method. After applying various kinds of perturbations to the humanoid during gait motion, the counteracting motion could be properly calculated. Although we 


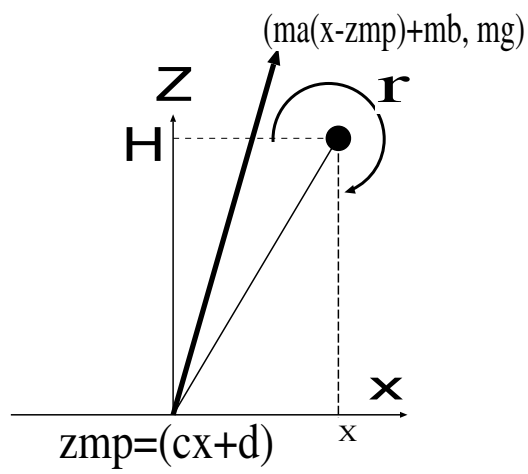

Fig. 1. The Angular Momentum inducing inverted Pendulum Mode (AMPM). The ZMP is allowed to move over the ground, and its position must be linearly dependent to that of the COM. The horizontal component of the ground force vector is allowed to change, by an amount which must be linearly dependent on the COM.

have applied the proposed method only to gait motion, the idea can be used for motions such as running and standing, as well.

\section{Angular Momentum INDUCing INVERTED PENDULUM MODEL}

In this section, we review the Angular Momentum inducing inverted Pendulum Model (AMPM) [8]. The AMPM enhances the 3DLIPM in the following directions; (1) the $\mathrm{ZMP}$ is allowed to move over the ground, (2) the ground force vector is calculated to be not only parallel to the vector connecting the ZMP and the COM; its horizontal element can be linearly correlated to the ZMP-COM vector (Fig. 1). As a result, rotational moment will be generated by the ground force. Let us assume the position of the COM is $(x, H)$, the position of the ZMP is $(c x+d, 0)$, and the vector of the ground force is parallel to the vector $(a(x-z m p)+b, g)$ where $a, b, c, d$ are constant values and $g$ is the gravity constant. As the height of the COM is assumed to have a constant value $H$, the relationship between the acceleration of the COM and its position can be written by:

$$
\begin{aligned}
F_{x}: F_{z} & =\ddot{x}:(\ddot{z}+g) \\
& =\frac{H}{g}(a(x-(c x+d))+b): H .
\end{aligned}
$$

The differential equation of the COM can then be written by the following form:

$$
\ddot{x}=a(1-c) x+b-a d .
$$

The explicit solution for this differential equation can be written as

$$
x=-\frac{b-a d}{a(1-c)}+C_{1} e^{-(\sqrt{a-a c}) t}+C_{2} e^{(\sqrt{a-a c}) t}
$$

where $C_{1}, C_{2}$ are constant values. As initial parameter values are set as $x=x_{0}$ and $\dot{x}=v_{0}$ at $t=0$, the constant values
$C_{1}, C_{2}$ will be as follows:

$$
\begin{aligned}
& C_{1}=\frac{1}{2}\left(x_{0}-\frac{v_{0}}{\sqrt{(a-a c)}}+\frac{b-a d}{a(c-1)}\right), \\
& C_{2}=\frac{1}{2}\left(x_{0}+\frac{v_{0}}{\sqrt{(} a-a c)}+\frac{b-a d}{a(c-1)}\right) .
\end{aligned}
$$

Then, the ground force vector can be written as

$$
\begin{aligned}
F_{x} & =m \ddot{x} \\
& =m(a-a c)\left(C_{1} e^{-(\sqrt{a-a c}) t}+C_{2} e^{(\sqrt{a-a c}) t}\right) \\
F_{z} & =m g
\end{aligned}
$$

where $m$ is the mass of the system. The rotational moment $r$ around the $y$-axis can be calculated by

$$
\begin{aligned}
r & =m(1-c)(a H-g)\left(C_{1} e^{-(\sqrt{a-a c}) t}+C_{2} e^{(\sqrt{a-a c}) t}\right) \\
& +m g\left(\frac{b}{a}\right)
\end{aligned}
$$

and the angular momentum $\omega_{t_{1}, t_{2}}$ generated by the rotational momentum between times $t=t_{1}, t_{2}$ can be obtained as

$$
\begin{gathered}
\omega_{t_{1}, t_{2}}=\left[\frac{m(1-c)(a H-g)}{\sqrt{a-a c}}\left(-C_{1} e^{-(\sqrt{a-a c}) t}+C_{2} e^{(\sqrt{a-a c}) t}\right)\right. \\
\left.+m g t\left(\frac{b}{a}\right)\right]_{t_{1}}^{t_{2}}+\omega_{1}
\end{gathered}
$$

where $\omega_{1}$ is the angular momentum at $t=t_{1}$.

\section{A. Using the AMPM to counteract external perturbation in the sagittal plane}

Suppose the motion of the humanoid in sagittal plane is defined as shown in Figure 2. To clarify the concept of our approach, let us assume here that no angular momentum around the COM is generated in the original feedforward motion. That means the ground force vector always passes through the COM. Let us assume the humanoid is first in single support phase, and external perturbation was applied to the humanoid body causing sudden increase in the linear and angular momentum when the COM is at Point A. The increased linear momentum can be reduced by using existing approaches of 3DLIPM. However, it was difficult to reduce the induced angular momentum by previous approaches especially when the amount is large.

The increase in the linear and angular momentum are defined here by $\Delta L$ and $\Delta M$, as well. Even after the perturbation, we assume the height of the center of gravity is same as before, and the vertical velocity of the center of gravity is zero as well. Actually, it is possible to summarize all the effect of the external perturbation to the increase in the horizontal component of the linear momentum and the angular momentum, by forcing the COM to stay at the same height using conventional feedback algorithm such as PD control, although this would further increase the angular momentum of the body around the center of gravity. After the 




Fig. 2. The gait motion pattern in the sagittal plane. The black dot represents the COM and the triangles on the bottom represent the support feet. The external perturbation is applied during single support phase, when the COM is at Point A. The positions of the COM when the following double support, single support, and double support phase start are defined here by Point $\mathrm{B}, \mathrm{C}$, and C', as well. The angular momentum generated by the external perturbation is compensated mainly between Point B and C'.

perturbation, the COM will move along the horizontal axis, and the ground force vector will penetrate the COM as same as in the original motion. Therefore, the angular momentum will stay at the same value during the single support phase. In order to reduce the increased linear and angular momentum to zero, the motion after the perturbation will be re-planned. The following strategies are used to counteract the increased momentum:

- the position the swing leg lands onto the ground will be modified

- rotational momentum will be applied to the body during the double support phase to counteract the angular momentum induced by the external perturbation.

For the motion during double support phase, the following two assumptions are made: (1) the coordinate values of Point $\mathrm{B}$ and $\mathrm{C}$ in Figure 2, which are the points of COM when the double support phase begins and ends, as well, will be the same as those in the original gait motion, and (2) The motion of the COM and the trajectory of the angular momentum will follow the rules of AMPM. The ground force vector will be parallel to the vector connecting the ZMP and COM at Point C. The acceleration of the COM will be uncontinuous at Point $\mathrm{B}$, as the ground force vector will be adjusted so that the angular momentum will be reduced to zero when the COM arrives to $\mathrm{C}$. Let us assume the position of the new foot position is decided and the coordinate value of the ZMP at Point $\mathrm{C}$ and $\mathrm{C}^{\prime}$ are defined by $z_{c}$ and $z_{c}^{\prime}$, as well. The new differential equation of the COM during the double support phase is defined here by

$$
\ddot{x}=p x+q
$$

where $p$ and $q$ are the parameters which are to be calculated. The condition that the increased angular momentum will be reduced to zero can be written by the following form:

$$
\omega_{B, C}=-\Delta M
$$

where $\omega_{B, C}$ is the angular momentum generated during double support which can be explicitly written by the form in Equation 3. As the ground force vector is parallel to the vector connecting the $\mathrm{ZMP}$ and $\mathrm{COM}$ at Point $\mathrm{C}$, the following equation must be satisfied:

$$
\frac{g}{H} x_{c}=p x_{c}+q
$$

where $x_{c}$ is the coordinate value of the COM at Point $\mathrm{C}$ and $H$ is the height of the COM.

By substituting Equation 6 into 5, the following equation can be obtained:

$$
\omega_{B, C}(p)=-\Delta M
$$

where $\omega_{B, C}(p)$ is a function that returns the angular momentum generated between Point $\mathrm{B}$ and Point $\mathrm{C}$ using $p$ as an input. Unfortunately, there is no explicit solution for $p$ in Equation 7. Although the solution must be calculated numerically, as the relationship between $p$ and $\omega_{B, C}(p)$ is linear around the solution, a high-precision solution can be obtained by limited number of iterations.

The increased linear momentum $\Delta L$ must also be reduced to zero. In order to do this, the method proposed by Kajita et al[5], which is to minimize the following function is used:

$$
\left(x_{c^{\prime}}-x_{c^{\prime}}^{O}\right)^{2}+\left(v_{c^{\prime}}-v_{c^{\prime}}^{O}\right)^{2}
$$

where $x_{c^{\prime}}$ and $v_{c^{\prime}}$ are the position and velocity of the COM at Point $C^{\prime}$ and $x_{c^{\prime}}^{0}$ and $v_{c^{\prime}}^{0}$ are the corresponding values in the original feedforward motion. The linear momentum is mostly compensated during the single support phase, especially when the support foot is in front of the COM.

To summarize, the motion in the frontal plane is calculated by searching for the foot-landing position that minimize Equation 8. The motion during the double support phase is determined by solving for $p$ using Equation 7 .

One problem remains here; although the angular momentum can be reduced to zero by adjusting the AMPM parameters, the posture of the body will remain different from the original gait motion, unless angular momentum that brings back the body to the original posture is induced. In order to solve this problem, we introduce a new criteria called difference of inertia, which can be used to estimate the amount of additional angular momentum that must be added to the body to recover the original posture. The difference of inertia can be defined as follows:

$$
\Delta I=\sum_{i}\left(c_{i}-c_{g}\right) \times\left(c_{i}-c_{i}^{o}\right)+R_{i} I_{i} R_{i}^{T}\left(\theta_{i}^{o}-\theta_{i}\right)
$$

where $c_{i}$ is the position of COM, $c_{i}^{o}$ is the COM in the original motion, $\theta_{i}$ is the orientation, $\theta_{i}^{o}$ is the orientation in the original motion, $R_{i}$ is the $3 \times 3$ rotational matrix, $I_{i}$ 
is the moment of inertia of segment $i$, as well, and $c_{g}$ is the COM of the whole body. By dividing the difference of inertia by the interval for the transition, it is possible to calculate the angular momentum needed. In order to recover the original motion inertia during double support phase, an angular momentum of value $\Delta I / \widetilde{T_{B, C}}$ must be added to the body, where $\widetilde{T_{B, C}}$ is the estimated duration of the double support phase which can be calculated by dividing the distance between $\mathrm{B}$ and $\mathrm{C}$ by the velocity of the COM at Point B:

$$
\widetilde{T_{B, C}}=\frac{x_{C}-x_{B}}{\dot{x}_{B}}
$$

where $x_{B}, \dot{x_{B}}$, and $x_{C}$ are the position and velocity of the $\mathrm{COM}$ at point $\mathrm{B}$, and the position of Point $\mathrm{C}$, as well.

Instead of solving for $p$ using Equation 7, the following equation can be used to calculate the motion to recover the original posture:

$$
\omega_{B, C}=-\Delta M+\beta \frac{\Delta I}{\widetilde{T_{B, C}}}
$$

where $\beta$ is a weight value smaller than 1 which is necessary for stable convergence.

For each of the following walking step, the motion for the next double support phase is recalculated using the error of the linear and angular momentum at the end of the previous double support phase, by solving Equation 10. For the difference of inertia, the value at the end of the previous double support phase is used. As a result, the motion gradually returns back to the original motion after a few steps.

\section{USING THE AMPM TO COUNTERACT EXTERNAL PERTURBATION IN THE FRONTAL PLANE}

The motion of the COM in the frontal plane can be explained by AMPM as shown in Figure 3. During double support phase, the ZMP will move proportionally to the COM, and during single support phase, the ZMP will stay at the same position under the supporting foot. If we assume no angular momentum is generated, the vector connecting the COM and the ZMP will always be parallel to the ground reaction force.

To clarify the idea of the feedback approach in this study, again, let us assume that the ground force vector is parallel to the vector connecting the COM and the $\mathrm{ZMP}$ in the original feedforward motion. External perturbation is applied to the body at Point A, during single support phase, as shown in Figure 3, and as a result, angular momentum of amount $\Delta M^{f}$ is induced around the frontal axis. In order to reduce this angular momentum to zero, the motion during the following double support phase and the single support phase will be modified. Just as same as in the motion in the sagittal plane, the following strategies are adopted: (1) the position the swing leg landing onto the ground will be changed (2) rotational momentum will be applied to the body during the

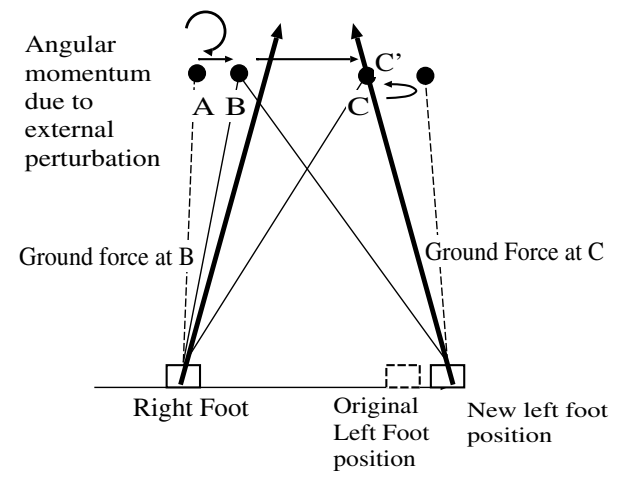

Fig. 3. Counteracting the angular momentum induced by external perturbation in the frontal plane. External perturbation is applied during single support phase at Point A. The position of the foot landing on the ground will be changed, and the ground force vector at Point $\mathrm{C}$ will be changed, as well. The ground force vector during the rest of the trajectories will be calculated in a way that the acceleration is continuous. The motion of the double support phase will be calculated in a way that when the right foot lands onto the ground after the single support by the left foot (Point C'), the position and velocity of the COM will be close to those of the original feedforward motion, and the angular momentum is reduced to zero.

double support phase to counter act the angular momentum induced by the external perturbation.

The new differential equation of the COM during the double support phase is defined here by

$$
\ddot{y}=p_{y} y+q_{y}
$$

where $p_{y}, q_{y}$ are AMPM parameters. As the duration of the double support phase, $T_{B, C}$ is determined by the motion in the sagittal plane, the position and velocity at point $C$ can be obtained by Equation 2 and its derivative:

$$
\begin{aligned}
y_{C} & =\frac{\sqrt{p_{y}}\left(y_{B}+\frac{d}{c}-\dot{y}_{B}\right)}{2} e^{-\sqrt{p_{y}} T_{B}, C} \\
& +\frac{\sqrt{p_{y}}\left(y_{B}+\frac{q_{y}}{p_{y}}+\dot{y_{B}}\right)}{2} e^{\sqrt{p_{y}} T_{B, C}}-\frac{q_{y}}{p_{y}} \\
\dot{y_{C}} & =\frac{p_{y}\left(y_{B}+\frac{p_{y}}{q_{y}}-\dot{y_{B}}\right)}{2} e^{-\sqrt{p_{y}} T_{B}, C} \\
& +\frac{p_{y}\left(y_{0}+\frac{d}{c}+\dot{y_{B}}\right)}{2} e^{\sqrt{p_{y}} T_{B}, C} .
\end{aligned}
$$

where $y_{B}, y_{C}, \dot{y_{B}}, \dot{y_{C}}$ are the positions and velocities of $\mathrm{COM}$ at Point $\mathrm{B}$ and Point $\mathrm{C}$, as well. The calculation done here is quite similar to those done for the motion in the sagittal plane. To calculate $p_{y}$ and $q_{y}$, the following two constraints are taken into account:

$$
\begin{aligned}
& \omega_{d}=-\Delta M^{f}+\Delta I^{f^{\prime}} \\
& \frac{y_{c}-z_{c}^{f}}{H} g=p_{y} y_{c}+q_{y}
\end{aligned}
$$

where $\Delta I^{f^{\prime}}$ is the difference of inertia at Point $\mathrm{B}$, and $\alpha$ is a constant value smaller than 1 to stabilize the convergence of the method. 


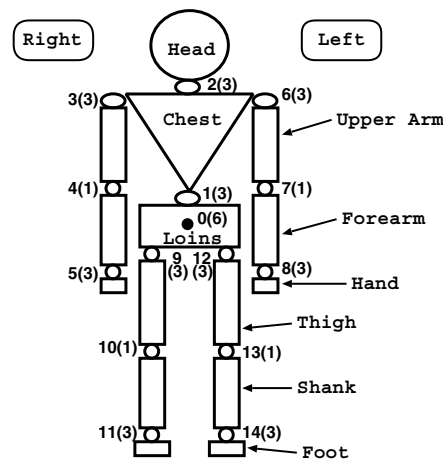

Fig. 4. The human body model used in this study. The number at the joints represents the joint's ID and their DOF.

For $z_{c}^{f}$, the position the foot lands onto the ground, a value that minimize the following mean square error function is adopted:

$$
\left(y_{c^{\prime}}-y_{c^{\prime}}^{O}\right)^{2}+\left(\dot{y}_{c^{\prime}}-\dot{y}_{c^{\prime}}^{O}\right)^{2}
$$

where $y_{c^{\prime}}$ and $\dot{y}_{c^{\prime}}$ are the position and velocity of the COM at Point $\mathrm{C}^{\prime}$ and $y_{c^{\prime}}^{0}$ and $y^{0}{ }_{c^{\prime}}$ are the corresponding values in the original feedforward motion.

To summarize, the motion in the frontal plane is calculated by searching for the foot-landing position that minimize Equation 14. The motion during the double support phase is determined by calculating the AMPM parameters $p_{y}$ and $q_{y}$ by using Equation 12 and 13 as constraints.

\section{Calculating the Generalized CoOrdinates USING INVERSE KINEMATICS}

As we have already defined the trajectories of the COM and the angular momentum, the next step is to calculate kinematic parameters that satisfy these constraints. Inverse kinematics is used for this purpose. A human body model with 42 degrees of freedom, as shown in Figure 4, was used. It should be noted that one translational degrees of freedom of is added to each knee to avoid the singularity due to its full extension. Trajectories of generalized coordinates of the human body model are defined here as $\boldsymbol{q}(t)=$ $\left(q_{1}(t), q_{2}(t), . ., q_{\mathrm{dof}}(t)\right)^{T}$ where dof is the number of degrees of freedom of the human body model, and the value is 42 . Generalized coordinates $\boldsymbol{q}(t)$ include the position and rotation of the root of the body in the 3D world coordinate system.

The relationship between velocity of the COM and velocity of the generalized coordinates can be written as follows:

$$
\dot{\boldsymbol{x}}_{g}=J_{\mathrm{com}} \dot{\boldsymbol{q}}
$$

where $J_{\text {com }}$ is the Jacobian matrix that consists of the partial derivatives of the COM by the generalized coordinates. Then, the acceleration of the COM can be derived as follows:

$$
\ddot{\boldsymbol{x}}_{g}=J_{\mathrm{com}} \ddot{\boldsymbol{q}}+\dot{J}_{\mathrm{com}} \dot{\boldsymbol{q}} .
$$

The angular momentum $\boldsymbol{r}$ and the first derivative of the generalized coordinates have a linear correlation:

$$
\boldsymbol{r}=R \dot{\boldsymbol{q}}
$$

The derivative of the angular momentum can be derived as follows:

$$
\dot{\boldsymbol{r}}=R \ddot{\boldsymbol{q}}+\dot{R} \dot{\boldsymbol{q}}
$$

The translational and rotational acceleration of the feet can be expressed as functions of $\ddot{\boldsymbol{q}}$ as well:

$$
\left(\ddot{\boldsymbol{p}}_{l}, \ddot{\boldsymbol{p}}_{r}, \ddot{\boldsymbol{\theta}}_{l}, \ddot{\boldsymbol{\theta}}_{r}\right)^{T}=J_{f} \ddot{\boldsymbol{q}}+\dot{J}_{f} \dot{\boldsymbol{q}}
$$

The trajectories of the feet are calculated by scaling the trajectories of the original feet using the new position of the foot steps;

$$
\begin{array}{r}
\left(p_{x}, p_{y}\right)= \\
\left(\frac{s_{x}^{i+1}-s_{x}^{i}}{s_{x, 0}^{i+1}-s_{x, 0}^{i}}\left(p_{x}^{0}-s_{x}^{i}\right)+s_{x}^{i}, \frac{s_{y}^{i+1}-s_{y}^{i}}{s_{y, 0}^{i+1}-s_{y, 0}^{i}}\left(p_{y}^{0}-s_{y}^{i}\right)+s_{y}^{i}\right)
\end{array}
$$

where $\left(s_{x}^{i}, s_{y}^{i}\right)$ and $\left(s_{x}^{i+1}, s_{y}^{i+1}\right)$ are the position of the $i$ th and $(i+1)$ th footsteps on the floor in the newly generated motion, $\left(s_{x, 0}^{i}, s_{y, 0}^{i}\right)$ and $\left(s_{x, 0}^{i+1}, s_{y, 0}^{i+1}\right)$ are the corresponding position of the footsteps in the original motion, and $\left(p_{x}, p_{y}\right)$ is the position of the foot in the horizontal plane in the newly generated motion, and $\left(p_{x}^{0}, p_{y}^{0}\right)$ is the corresponding position in the original motion.

The rotation of the feet in the new motion will be calculated by using the step length as a scaling factor: $\theta_{y}=$ $\left(s_{l} / s_{l}^{0}\right) \theta_{y}^{0}$. This is due to the fact that the orientation of the feet enlarges as the step length gets larger.

Combining Equation 15, 16, and 17, linear constraints that must be satisfied by the body can be summarized to the following form:

$$
\boldsymbol{\lambda}=J_{\mathrm{all}} \ddot{\boldsymbol{q}}+\dot{J}_{\mathrm{all}} \dot{\boldsymbol{q}} .
$$

where $\boldsymbol{\lambda}=\left(\ddot{\boldsymbol{x}}_{g}, \dot{\boldsymbol{r}}, \ddot{\boldsymbol{p}}_{l}, \ddot{\boldsymbol{\theta}}_{l}, \ddot{\boldsymbol{p}}_{r}, \ddot{\boldsymbol{\theta}}_{r}\right)^{T}$, and $J_{\text {all }}=$ $\left(J_{\text {com }}, R, J_{f}\right)^{T}$. Calculating $\ddot{\boldsymbol{q}}$ that satisfies Equation 18 can be considered as an inverse kinematics problem.

Since the goal is to calculate a stable gait motion, the following quadratic form is minimized with respect to $\ddot{q}$ :

$$
\left(\ddot{\boldsymbol{q}}-k\left(\boldsymbol{q}-\boldsymbol{q}_{0}\right)+d \cdot \dot{\boldsymbol{q}}\right)\left(\ddot{\boldsymbol{q}}-k\left(\boldsymbol{q}-\boldsymbol{q}_{0}\right)+d \cdot \dot{\boldsymbol{q}}\right)^{T} .
$$

where $k, d$ are the elastic and damping constants, respectively. Quadratic programming is used to calculate $\ddot{\boldsymbol{q}}$ by minimizing Equation 19 subject to the constraint given by Equation 18.

Using the calculated acceleration, the values of thegeneralized coordinates and their velocity were updated step by step, and finally, the whole trajectory was obtained. 


\section{EXPERIMENTS}

The motion of the human body was first generated by planning the motion of the COM using AMPM without any angular momentum around the COM. After the trajectories of the COM and the feet were determined, the trajectories of the joint angles were calculated using inverse kinematics. This motion is shown in Figure 5.

Then, while the humanoid is performing this feedforward motion, external perturbation was applied to the body during the single support phase. Two experiments were done and in each of them different level of impact was applied to the body. In the first experiment, a weaker impact that induced additional linear momentum of $0.1 \mathrm{~kg} \cdot \mathrm{m} / \mathrm{s}$ and angular momentum of $12.0 \mathrm{~kg} \cdot \mathrm{m}^{2} / \mathrm{s}$ around the COM. In the second experiment, stronger perturbation was applied, which induced additional linear momentum of $0.2 \mathrm{~kg} \cdot \mathrm{m} / \mathrm{s}$ and angular momentum of $24.0 \mathrm{~kg} \cdot \mathrm{m}^{2} / \mathrm{s}$

First, gait motions that only counteract the external perturbation but do not recover the original postures were calculated. The results are shown in Figure 6 (weak perturbation) and 7 (strong perturbation), as well. Although the humanoid can stop the rotation of the chest, the chest remains bent for the following motion.

Next, the feedback criteria that takes into account the difference of inertia was used. Again, two experiments were done. In each of them different level of perturbation was applied and the same additional pair of linear and angular momentum (linear momentum of $0.1 \mathrm{~kg} \cdot \mathrm{m} / \mathrm{s}$ and angular momentum of $12.0 \mathrm{~kg} \cdot \mathrm{m}^{2} / \mathrm{s}$ by the weaker impact, and linear momentum of $0.2 \mathrm{~kg} \cdot \mathrm{m} / \mathrm{s}$ and angular momentum of $24.0 \mathrm{~kg} \cdot \mathrm{m}^{2} / \mathrm{s}$ by the stronger impact) were induced. After the perturbation, the thorax rotates to the front due to the increased angular momentum. This angular momentum is counteracted during the following double support phase, and the original gait motion is gradually recovered after a few steps. The trajectories of the two motions are shown in Figure 8 and 9, as well. In both of the motions, after stopping the rotation of the chest, the body is brought back to the original upright posture.

The trajectories of the angular momentum around the lateral axis when the difference of inertia is taken into account (dashed line) and when it is not taken into account (solid line) are plotted in Figure 10. Without including the difference of inertia in the criteria function, the angular momentum just decreases to zero. On the other hand, when this information is used for feedback control, after the value decreases to zero, negative angular momentum that is needed to move the body back to the upright posture is generated, and gradually converges to zero after a few steps.

\section{DISCUSSION}

As the gait motion is much more unstable during the single support phase than during the double support phase,

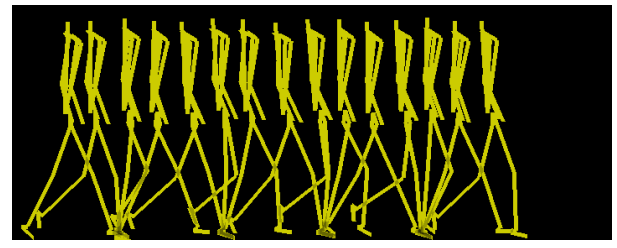

Fig. 5. The original gait motion of the humanoid gait in the sagittal plane generated using AMPM.

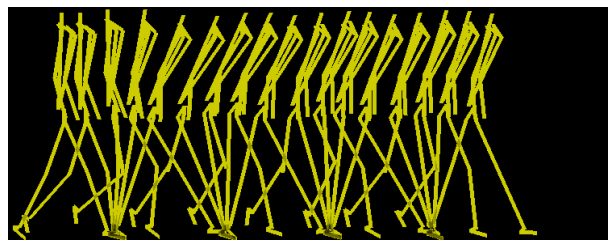

Fig. 6. The trajectory of the humanoid walking in the sagittal plane. Assuming external perturbation has induced additional linear and angular momentum $\left(0.1 \mathrm{~kg} \cdot \mathrm{m} / \mathrm{s}\right.$ and $\left.12.0 \mathrm{~kg} \cdot \mathrm{m}^{2} / \mathrm{s}\right)$ during the single support phase, the increased angular momentum is counteracted during the following double support and single support phases.

we assume here the external perturbation happened during the single support phase.

As the stance distance is much longer along the anterior axis than along the lateral axis, the method in this study is more effective for counteracting angular momentum around the lateral axis than around the frontal axis. Strong interference along the lateral axis will greatly affect the velocity of the COM and then the humanoid will have to step out the next step lateral to the supporting foot. In such case, however, the swing foot can easily collide with the supporting leg unless precise path planning is done for the landing motion. This would be the next subject to be solved.

The inertia criteria shows good performance to bring the current posture to the target posture. When calculating the motion using inverse kinematics by using the angular momentum and linear momentum as constraints as constraints, the obtained motion gradually deviates from the target motion even though the objective function of inverse kinematics include terms based on the difference of the target posture

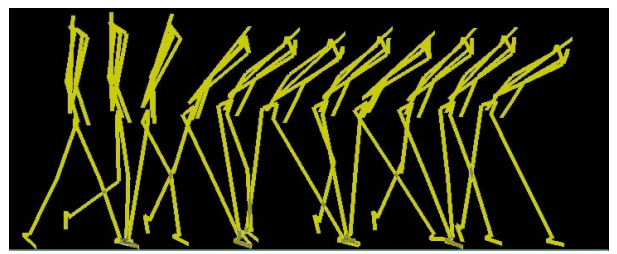

Fig. 7. The trajectory of the humanoid walking in the sagittal plane. Assuming external perturbation has induced additional linear and angular momentum $\left(0.2 \mathrm{~kg} \cdot \mathrm{m} / \mathrm{s}\right.$ and $\left.24.0 \mathrm{~kg} \cdot \mathrm{m}^{2} / \mathrm{s}\right)$ during the single support phase, the increased angular momentum is counteracted during the following double support and single support phases. 


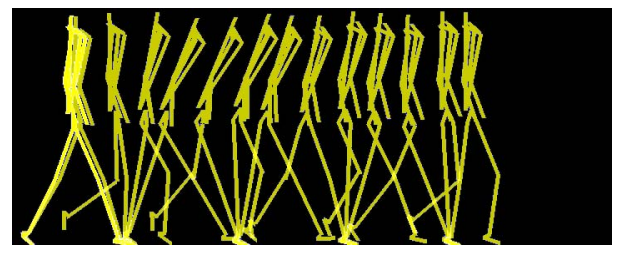

Fig. 8. The motion of the humanoid gait in the sagittal plane. After 0.1 seconds in the initial single support phase, additional linear and angular momentum around the center of gravity is added to the body $(0.1 \mathrm{~kg}$. $\mathrm{m} / \mathrm{s}$ and $12.0 \mathrm{~kg} \cdot \mathrm{m}^{2} / \mathrm{s}$ ). The feedforward motion is reorganized so that the increased angular momentum can be counteracted during the following double support and single support phase.

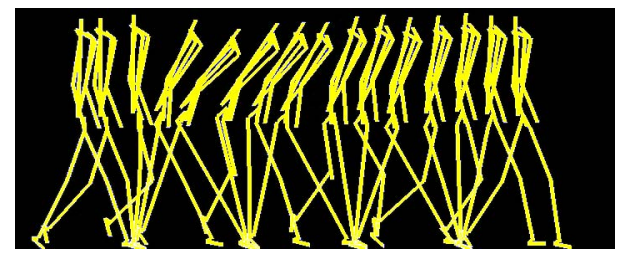

Fig. 9. The motion of the humanoid gait in the sagittal plane. After 0.1 seconds in the initial single support phase, additional linear and angular momentum around the center of gravity is added to the body $(0.2 \mathrm{~kg}$. $\mathrm{m} / \mathrm{s}$ and $24.0 \mathrm{~kg} \cdot \mathrm{m}^{2} / \mathrm{s}$ ). The feedforward motion is reorganized so that the increased angular momentum can be counteracted during the following double support and single support phase.

and the current posture. In such case, it is necessary to add additional angular momentum to the body in order to restore the original posture. By adding a term based on inertia criteria to the target angular momentum constraint, it is possible to stabilize the posture to the target posture.

\section{SUMMARY AND FUTURE WORK}

In this paper, we proposed a new method for biped humanoids to counteract a large amount of angular momentum induced by strong external perturbations applied to the body during gait motion. Such angular momentum can easily cause the humanoid to fall down onto the ground. We use AMPM, which is an enhanced version of 3DLIPM, to calculate the counteracting motion in real-time.

Although we assumed large amount of force as the external perturbation, the stepping patterns and the position of the foot landing to the ground were kept the same as the original feedforward motion. It is, however, not possible to counteract very strong perturbation especially in the lateral direction if the stepping pattern and the landing positions are also reorganized. Calculating new patterns of the appropriate stepping parameters will be the next goal to be achieved.

\section{ACKNOWLEDGEMENT}

The work described in this paper was partially supported by a grant from City University of Hong Kong (Project No. 7100293).

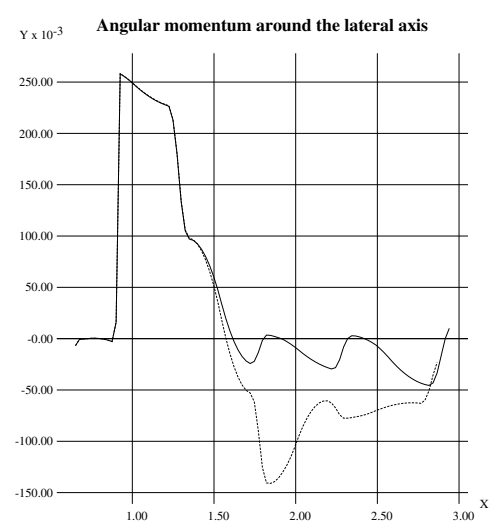

Fig. 10. The trajectory of the angular momentum around the lateral axis when the AMPM is used only to counteract the angular momentum generated by the external perturbation (solid line), and when further angular momentum is generated for feedback motion to turn the motion back to the original gait

\section{REFERENCES}

[1] Yasutaka Fujimoto and Atsuo Kawamura. Robust biped walking with active interation control between robot and environment. Proceedings of IEEE AMC96, 1996.

[2] M. Fujita, Y. Kuroki, T. Ishida, and T. Doi. Autonomousbehavior control architecture of entertainment humanoidrobot sdr-4x. Proceedings of the 2003 IEEE/RSJ In-ternational Conference on Intelligent Robots and Systems(IROS 03), pages 960-967, 2003.

[3] K Hirai, M Hirose, Y Haikawa, and T. Takenaka. The development of honda humanoid robot. Proceedings of IEEE International Conference on Robotics and Automation, pages 1321-1326, 1998.

[4] Shuuji Kajita, Fumio Kanehiro, Kenji Kaneko, Kiyoshi Fujiwara, Kensuke Harada, Kazuhito Yokoi, and Hiroshi Hirukawa. Resolved momentum control: humanoid motion planning based on the linear and angular momentum. Proceedings of IEEE/RSJ International Conference on Intelligent Robots and Systems, pages 1644-1650, 2003.

[5] Shuuji Kajita, Osamu Matsumoto, and Muneharu Saigo. Real-time $3 \mathrm{~d}$ walking pattern generation for a biped robot with telscopic legs. Proceedings of the 2001 IEEE International Conference on Robotics and Automation, 2001.

[6] Shuuji Kajita, Tomio Yamaura, and Akira Kobayashi. Dynamic walking control of a biped robot along a potential energy conserving orbit. IEEE Transactions on Robotics and Automation, 8(4), 1992.

[7] Shuuji Kajita, Kazuhito Yokoi, Muneharu Saigo, and Kazuo Tanie. Balancing a humanoid robot using backdrive concerned torque control and direct angular momentum feedback. Proceedings of IEEE International Conference on Robotics and Automation, 2001.

[8] Shunsuke Kudoh and Taku Komura. C $\hat{2}$ continuous gait-pattern generation for biped robots. Proceedings of IEEE/RSJ International Conference on Intelligent Robots and Systems, pages 1644-1650, 2003.

[9] Qinghua Li, Atsuo takanishi, and Ichiro Kato. Learning control of compensative trunk motion for biped walking robot based on $\mathrm{zmp}$. Proceedings of the 1992 IEEE/RSJ Intl. Conference on Intelligent Robot and Systems, pages 597-603, 1992.

[10] Shigeki Nakaura Napoleon and Mitsuji Sampei. Balance control analysis of humanoid robot based on zmp feedback control. Proceedings of IEEE/RSJ International Conference on Intelligent Robots and Systems, 2002.

[11] Koichi Nishiwaki, Tomomichi Sugihara, Satoshi Kagami, Masayuki Inaba, and Hirochika Inoue. Online mixture and connection of basic motions for humanoid walking control by footprint specification. IEEE International Conference on Robotics and Automation, pages 4110 4115, 2001. 